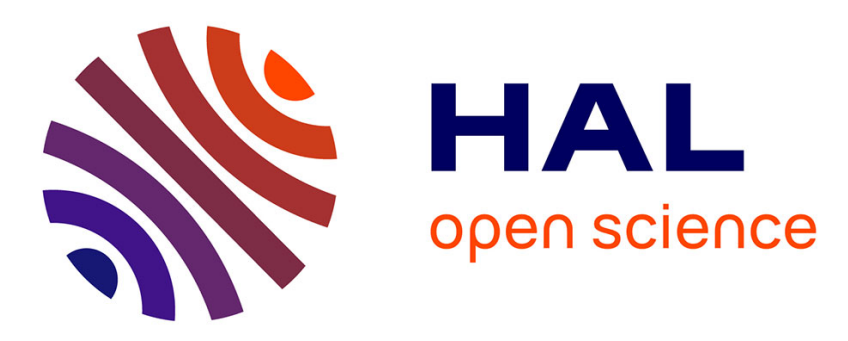

\title{
An Integral Face Formulation for Thin Non-Conductive Magnetic Regions
}

G. Chavin-Collin, B. Bannwarth, D. Cavallera, Olivier Chadebec, N. Galopin, Gérard Meunier, L.-L. Rouve

\section{- To cite this version:}

G. Chavin-Collin, B. Bannwarth, D. Cavallera, Olivier Chadebec, N. Galopin, et al.. An Integral Face Formulation for Thin Non-Conductive Magnetic Regions. IEEE Transactions on Magnetics, 2019, 55 (6), 10.1109/TMAG.2019.2895959 . hal-02350902

\section{HAL Id: hal-02350902 https://hal.science/hal-02350902}

Submitted on 6 Nov 2019

HAL is a multi-disciplinary open access archive for the deposit and dissemination of scientific research documents, whether they are published or not. The documents may come from teaching and research institutions in France or abroad, or from public or private research centers.
L'archive ouverte pluridisciplinaire HAL, est destinée au dépôt et à la diffusion de documents scientifiques de niveau recherche, publiés ou non, émanant des établissements d'enseignement et de recherche français ou étrangers, des laboratoires publics ou privés. 


\title{
An Integral Face Formulation for Thin Non-Conductive Magnetic Regions
}

\author{
G. Chavin-Collin ${ }^{1,2}$, B. Bannwarth ${ }^{1}$, D. Cavallera ${ }^{2}$, O. Chadebec ${ }^{1}$, N. Galopin ${ }^{1}$, G. Meunier ${ }^{1}$ and L-L. Rouve $^{1}$ \\ ${ }^{1}$ Univ. Grenoble Alpes, CNRS, Grenoble INP, G2Elab, F-38000 Grenoble, France \\ ${ }^{2}$ Naval Group, 75732 Paris, France
}

\begin{abstract}
This article deals with the use of the Volume Integral Method (VIM) to compute the magnetic anomaly created by a non-conductive ferromagnetic thin shell placed in a static inductor field. An original facet integral formulation considering the magnetic induction as unknowns is presented. The use of thin shell element assumption leads to a surface mesh decreasing highly the number of elements so the computation cost. The method is very performant in terms of speed and accuracy.

Index Terms - Magnetostatic, volume integral method, thin shell element, shielding modeling.
\end{abstract}

\section{INTRODUCTION}

An alternative to the finite element method is to solve a magnetostatic problem with integral methods. The advantage is that it does not require the mesh of the air region reducing the number of degrees of freedom and improving the accuracy when the field in air has to be computed. The well-known drawback is the parabolic increase of the integration time but today, the emergence of matrix compression techniques has made this kind of methods very competitive.

An attractive field of application for integral formulations is the modeling of shieldings. Because these devices present a high ratio between air volume and active region volume, integral approaches are particularly relevant in such a context. Another very suitable application can be the calculation of a static magnetic anomaly created by navy ships.

A magnetostatic integral method based on first order facet elements interpolation of the magnetic flux density has already been proposed in [1]. A very similar formulation based on the edge interpolation of the magnetic vector potential can also be found in [2]. Unfortunately, both approaches have been developed for volume regions and cannot be simply applied to thin shell geometry because the thickness has to be meshed, leading to a huge number of elements.

In this paper, we propose an original formulation which is very efficient for the solving of problems associated to thin regions. The use of the thin shell element assumption introduced in the VIM proposed in [1] leads only to a 2D discretization of the medium surface of the ferromagnetic region, decreasing dramatically the number of degrees of freedom and saving computational time. The accuracy of the method is demonstrated by comparing results obtained with an analytical solution. A more complex problem is also solved and it is shown that the formulation is well adapted to the use of matrix compression techniques.

\section{MAGNetostatic INTEGRAL EQUATION}

Let us consider a magnetic problem composed of a ferromagnetic region with a relative permeability $\mu_{\mathrm{r}}$ and a static inductor magnetic field $\mathbf{H}_{\mathbf{0}}$ (Fig.1). For such problem, it is classical to assume that the magnetic material is linear and isotropic. It will be the case in the following. However, the proposed method is enough general to be easily extended to nonlinear and anisotropic cases. In the magnetic domain, the total field can be decomposed as a sum of an inductor field $\mathbf{H}_{\mathbf{0}}$ and a reduced field $\mathbf{H}_{\text {red }}$ due to the magnetization $\mathbf{M}$, which is the material's reaction to $\mathbf{H}_{\mathbf{0}} . \mathbf{H}_{\text {red }}$ derives from a scalar reduced potential $\varphi_{\text {red. }}$.

$$
\mathbf{H}(\mathbf{r})=\mathbf{H}_{\mathbf{0}}(\mathrm{r})+\mathbf{H}_{\text {red }}(\mathrm{r})=\mathbf{H}_{\mathbf{0}}(\mathrm{r})-\nabla \varphi_{\text {red }}(\mathrm{r})
$$
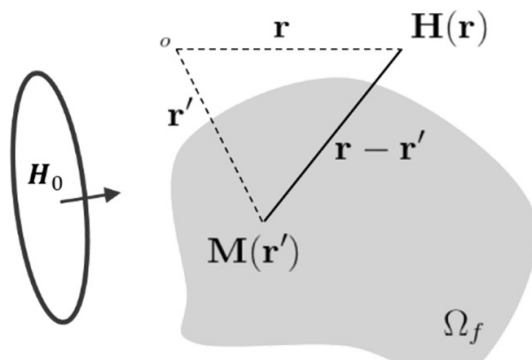

Fig. 1. Domain under study with ferromagnetic region $\Omega_{\mathrm{f}}$ and inductor field $\mathbf{H}_{\mathbf{0}}$.

As mentioned before, the ferromagnetic domain is associated to a linear behavior law.

$$
\mathbf{M}=\left(\mu_{\mathrm{r}}-1\right) \mathbf{H}=\chi \mathbf{H}
$$

The Helmholtz decomposition permits to write $\varphi_{\text {red }}$ as [3]:

$$
\varphi_{\text {red }}=\frac{1}{4 \pi} \int_{\Omega_{\mathrm{f}}} \frac{\mathbf{M} \cdot\left(\mathbf{r}-\mathbf{r}^{\prime}\right)}{\left|\mathrm{r}-\mathrm{r}^{\prime}\right|^{3}} \mathrm{~d} \Omega_{\mathrm{f}}
$$

Equations (1) and (2) combined to the integral expression of $\varphi_{\text {red }}$ leads to the magnetostatic integral equation with the magnetization $\mathbf{M}$ as state variable:

$$
\frac{\mathbf{M}}{\chi}+\frac{1}{4 \pi} \boldsymbol{\nabla} \int_{\Omega_{\mathrm{f}}} \frac{\mathbf{M} \cdot\left(\mathbf{r}-\mathbf{r}^{\prime}\right)}{\left|\mathrm{r}-\mathrm{r}^{\prime}\right|^{3}} \mathrm{~d} \Omega_{\mathrm{f}}=\mathbf{H}_{\mathbf{0}}
$$

A point matching method or a Galerkin projection can be used to solve directly this equation with the $\mathbf{M}$ components as degrees of freedom [3]. In such an approach, 0-order shape functions for each component are classically used. This formulation is known as Magnetic Moment Method (MMM) [3]. The MMM is able to give accurate results for simple problems but is not general and can lead to inaccuracies in some configurations. Others authors have proposed to solve (4) using more sophisticated approaches based on others quantities 
discretized on adequate functional spaces as unknowns. For instance, let us mention the scalar potential associated to nodal shape functions [4] or the magnetic field associated to edge shape functions [5].

In previous papers, it has been proposed to solve (4) choosing B as unknown [1] or even the magnetic vector potential [2]. However, these formulations were limited to volume magnetic regions and their use in the context of magnetic shielding is not efficient in terms of computation time. In this following article, the application of the $\mathbf{B}$-formulation to thin ferromagnetic sheets leading to surface meshes is presented.

\section{MAGNETOSTATIC INTEGRAL B-FORMULATION}

Let us consider the constitutive laws of the material with its reluctivity $v$ linking $\mathbf{B}$ to $\mathbf{M}$.

$$
\begin{gathered}
\mathbf{H}=v \mathbf{B} \\
\mathbf{M}=\left(v_{0}-v\right) \mathbf{B}
\end{gathered}
$$

According to (5) and (6) the integral equation (4) can be rewritten with $\mathbf{B}$ as unknown:

$$
v \mathbf{B}+\frac{1}{4 \pi} \boldsymbol{\nabla} \int_{\Omega_{\mathrm{m}}} \frac{\left(v_{0}-v\right) \mathbf{B} \cdot\left(\mathbf{r}-\mathbf{r}^{\prime}\right)}{\left|\mathrm{r}-\mathrm{r}^{\prime}\right|^{3}} \mathrm{~d} \Omega_{\mathrm{m}}=\mathbf{H}_{\mathbf{0}}
$$

This equation is solved with the unknown $\mathbf{B}$ interpolated by facet shape functions (Whitney 2-form) [1]. The advantage of using such an interpolation is to ensure the continuity of the normal component of the magnetic induction to the facet between two adjacent elements.

When volume elements have a small thickness with respect to their other dimensions and are associated to a high permeability, the assumption of the thin element can be used. It considers that the magnetic induction is tangential to the sheet and uniform across its thickness "t" [6] (Fig. 2).

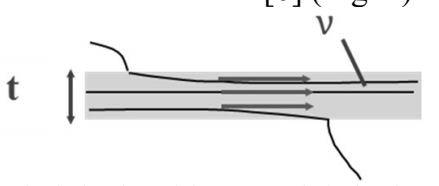

Fig. 2.Figure showing the behavior of the magnetic induction for a thin element: the induction is constant across the thickness and tangential to the surface.

Under this assumption, the magnetic flux density is chosen as a state variable and associated to degenerated facet functions on an equivalent surface mesh (often known as RaviartThomas basis functions). The number of unknowns is then decreased, all the normal components along the thickness being suppressed. A first order interpolation is used:

$$
\mathbf{B}=\sum_{\mathrm{f}=1}^{\mathrm{N}_{\mathrm{f}}} \boldsymbol{w}_{f} \frac{\Phi}{\mathrm{t}}=\sum_{\mathrm{f}=1}^{\mathrm{N}_{\mathrm{f}}} \boldsymbol{w}_{f} \Phi_{f}
$$

where $\mathrm{N}_{\mathrm{f}}$ is the number of facets (i.e. edges of the surface mesh), $\mathbf{w}_{f}$ the facet function pondering $\Phi_{f}$ which is the total flux flowing through the facet.

Besides, the following properties are verified:

$$
\begin{gathered}
\boldsymbol{w}_{f} \cdot \mathbf{n}_{f}=w_{n f}= \pm \frac{1}{\mathrm{l}_{f}} \\
\boldsymbol{\nabla} \cdot \boldsymbol{w}_{f}= \pm \frac{1}{\mathrm{~S}_{e}}
\end{gathered}
$$

where $l_{f}$ is the length of the facet and $S_{e}$ is the area of the element. The sign of (9) and (10) depends on the orientation of the facet " $f$ ".

Let us notice that these facet functions lead to a linear interpolation of $\mathbf{B}$ on each element of the surface mesh (Fig. 3).

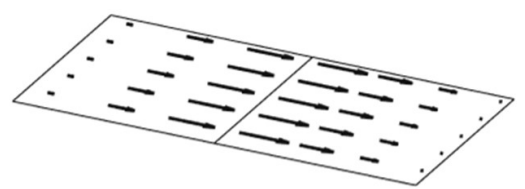

Fig. 3. A representation of $\mathbf{B}$ by facet functions on a surface mesh.

Equation (7) is discretized with (8) and a Galerkin projection using $\boldsymbol{w}_{g}^{\prime}$ (the same Raviart-Thomas function) is applied:

$$
\begin{gathered}
\sum_{f=1}^{N_{f}}\left(\int_{\Omega_{m}} v \boldsymbol{w}_{g}^{\prime} \cdot \boldsymbol{w}_{\boldsymbol{f}} d \Omega_{m}\right) \Phi \\
+\int_{\Omega_{m}} \boldsymbol{w}_{g}^{\prime} \cdot \boldsymbol{\nabla} \varphi_{\text {red }} d \Omega_{m}=\int_{\Omega_{m}} \boldsymbol{w}_{g}^{\prime} \cdot \boldsymbol{H}_{\mathbf{0}} d \Omega_{m}
\end{gathered}
$$

This can be written:

$$
R \Phi_{i n}+I=U_{0}
$$

with:

$$
\begin{gathered}
R_{\mathrm{ij}}=\frac{1}{\mathrm{t}} \int_{\Omega_{m}} \boldsymbol{v} \boldsymbol{w}_{i} \cdot \boldsymbol{w}_{j} d \Omega_{m} \\
U_{0 \mathrm{i}}=\int_{\Omega_{m}} \boldsymbol{w}_{i} \cdot \boldsymbol{H}_{\mathbf{0}} d \Omega_{m} \\
I_{\mathrm{i}}=\int_{\Omega_{m}} \boldsymbol{w}_{i} \cdot \boldsymbol{\nabla} \varphi_{\text {red }} d \Omega_{m}
\end{gathered}
$$

where $\boldsymbol{\Phi}_{\text {in }} \in \mathbb{R}^{\mathrm{N}_{\mathrm{f}} \times 1}$ is the internal flux flowing through the facet in the mesh, $\mathbf{R} \in \mathbb{R}^{\mathrm{N}_{\mathrm{f}} \times \mathrm{N}_{\mathrm{f}}}, \boldsymbol{I} \in \mathbb{R}^{\mathrm{N}_{\mathrm{f}} \times 1}$ and $\boldsymbol{U}_{\mathbf{0}} \in \mathbb{R}^{\mathrm{N}_{\mathrm{f}} \times 1}$. By applying the divergence theorem and properties (9) and (10), and by considering that fluxes flowing through the facet on the boundary of the domain are equal to zero, it comes:

$$
I_{\mathrm{i}}=\int_{\Omega_{m}} \boldsymbol{w}_{i} \cdot \boldsymbol{\nabla} \varphi_{\text {red }} d \Omega_{m}=\overline{\varphi_{\text {red }_{e 1}}}-\overline{\varphi_{\text {red }_{e 2}}}
$$

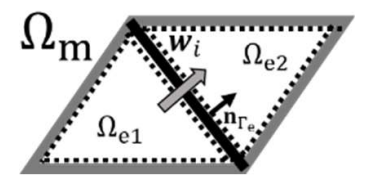

Fig. 4. Domain composed by 2 surface element " $\mathrm{e}_{1}$ " and " $\mathrm{e}_{2}$ " adjacent to a facet " $i$ " associated to the function $\boldsymbol{w}_{i}, \mathbf{n}_{\Gamma_{\mathrm{e}}}$ is the normal vector to the facet.

where $\overline{\varphi_{\text {red }} 1}$ and $\overline{\varphi_{\text {red }} 2}$ are the averaged reduced scalar potential on surface elements " $\mathrm{e}_{1}$ " and " $\mathrm{e}_{2}$ " adjacent to a facet "i". Let us notice that equation (16) can be seen as a difference of averaged reduced scalar potential value between elements inside the domain sharing a given facet.

Equation (12) can be interpreted as the matrix representation of an equivalent circuit composed, in this case, of a network of reluctances. In this circuit, each facet of the mesh is associated to a reluctance and each barycenter of the mesh elements to a circuit node. At this step, the vectors $\Phi_{f}$ and $I$ are unknown. It is necessary to take into account the flux going from the shell to the air in order to complete the system. 


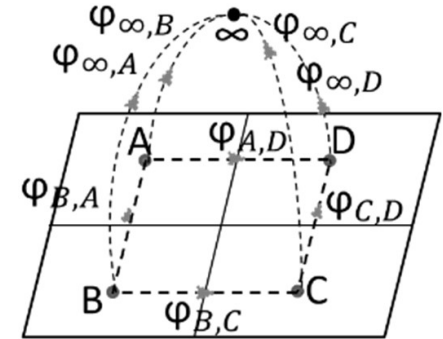

Fig. 5. Mesh composed by four face elements, it highlights the equivalent circuit with a node at the infinity.

To complete the circuit, a node is added at the infinite and its potential imposed (fixed) to zero (Fig. 5). The expression of the difference of averaged potential between a surface element and the infinite node is:

$$
\Delta \bar{\varphi}_{\text {red }} \rightarrow \infty=\int_{\mathrm{S}_{e}} \frac{\varphi_{\text {red }}}{\mathrm{S}_{e}} d \mathrm{~S}_{e}
$$

According to (3), the expression of the contribution of surface element e to the potential after applying divergence theorem is:

$$
\begin{aligned}
\varphi_{\text {red }}=-\sum_{\mathrm{e}=1}^{\mathrm{N}_{\mathrm{e}}} \frac{1}{4 \pi} \int_{\Gamma_{\mathrm{e}}}\left(\frac{\mathbf{M} \cdot \mathbf{n}_{\Gamma_{\mathrm{e}}}}{\left|\mathrm{r}-\mathrm{r}^{\prime}\right|}\right) d \Gamma_{\mathrm{e}} \\
\quad+\sum_{\mathrm{e}=1}^{\mathrm{N}_{\mathrm{e}}} \frac{1}{4 \pi} \int_{\mathrm{S}_{\mathrm{e}}} \frac{\boldsymbol{\nabla}_{s} \cdot \mathbf{M}}{\left|\mathrm{r}-\mathrm{r}^{\prime}\right|} \mathrm{dS}_{\mathrm{e}}
\end{aligned}
$$

where $\mathrm{N}_{\mathrm{e}}$ is the surface element number, $\Gamma_{\mathrm{e}}$ is the boundary of the element, $\mathbf{n}_{\Gamma_{\mathrm{e}}}$ is the normal vector going out from element $\mathrm{e}$ and tangential to the average surface (Fig. 4). Considering the reluctivity $v$ constant in the material, B.n is preserved as M.n, on the facet. The first terms of the integral (18) are canceled two by two on the mesh because the normal vectors are opposed. We get a new expression for the potential:

$$
\varphi_{\text {red }}=\frac{1}{4 \pi} \sum_{e=1}^{N_{e}} \int_{S_{e}} \frac{\left(v_{0-}-v\right) \boldsymbol{\nabla}_{s} . \mathbf{B}}{\left|\mathrm{r}-\mathrm{r}^{\prime}\right|} d S_{e}
$$

Let us notice that the surface divergence of $\mathbf{B}$, which is interpolated by facet shape functions, is constant per element. If $\mathbf{B}$ is replaced by the expression (8) in (19), a new equation is obtained from (17):

$$
\begin{gathered}
\Delta \overline{\boldsymbol{\varphi}_{\text {red }_{\infty}}}+\boldsymbol{L} \boldsymbol{\Phi}_{\infty}=0 \\
L_{i, j}=\frac{1}{4 \pi} \frac{\left(v_{0-} v_{j}\right)}{S_{e i} S_{e j}} \int_{S_{e i}} \int_{S_{e j}} \frac{1}{\left|\mathrm{r}-\mathrm{r}^{\prime}\right|} d S_{e i} d S_{e j}
\end{gathered}
$$

The final algebraic system is then:

$$
\left[\begin{array}{cc}
\mathbf{R} & 0 \\
0 & \mathbf{L}
\end{array}\right]\left[\begin{array}{l}
\boldsymbol{\Phi}_{\text {in }} \\
\boldsymbol{\Phi}_{\infty}
\end{array}\right]+\left[\begin{array}{l}
\Delta \overline{\boldsymbol{\varphi}_{\text {red }_{\text {in }}}} \\
\Delta \overline{\boldsymbol{\varphi}_{\text {red }_{\infty}}}
\end{array}\right]=\left[\begin{array}{c}
\mathbf{U}_{\mathbf{0}} \\
0
\end{array}\right]
$$

where $\boldsymbol{\Phi}_{\infty} \in \mathbb{R}^{\mathrm{N}_{\mathrm{e}} \times 1}$ is the vector of external fluxes flowing from each element to the air. Let us notice that $\mathrm{R}$ is a finite element matrix (sparse) while $L \in \mathbb{R}^{\mathrm{N}_{\mathrm{e}} \times \mathrm{N}_{\mathrm{e}}}$ is an integral matrix coupling all the elements of the mesh by the Green's function. This matrix is full but can be efficiently and easily compressed by standard compression techniques like Fast Multipole
Methods (FMM) or Adaptative Cross Approximation (ACA). $\mathbf{U}_{\mathbf{0}}$ can be easily obtained by the numerical integration of $\mathbf{H}_{\mathbf{0}}$ computed by Biot-Savart's law on the mesh.

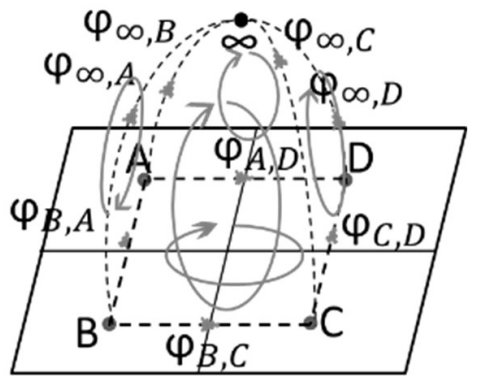

Fig. 6. This picture shows five loops for this mesh. In fact, only four loops are independent.

The final circuit equations is:

with:

$$
(R+L) \Phi+\Delta \overline{\varphi_{\text {red }}}=U_{0}
$$

$$
\Delta \overline{\varphi_{\text {red }}}=\left(\begin{array}{l}
\Delta \overline{\varphi_{\text {red }_{\text {in }}}} \\
\Delta \overline{\varphi_{\text {red }_{\infty}}}
\end{array}\right)
$$

It remains to ensure the free-divergence of the induction in the equivalent circuit. It can be achieved by finding a set of equivalent independent loops associated to the equivalent circuit (Fig.6). Classically in the domain of circuit analysis, we have:

$$
\mathbf{P} \Delta \overline{\varphi_{\text {red }}}=0
$$

where $P \in \mathbb{R}^{N_{B I} \times N_{f}}$ is the branch-fundamental independent loop incidence matrix (the value of each matrix element is $-1,0$ or 1 ) which links the flux of branches (i.e. facet) to independent loops fluxes. $\mathrm{N}_{\mathrm{BI}}$ is the number of independent fluxes. It comes:

$$
\boldsymbol{P}(\boldsymbol{R}+\boldsymbol{L}) \mathbf{P}^{t} \boldsymbol{\Phi}_{\text {loop }}=\boldsymbol{P} \boldsymbol{U}_{\mathbf{0}}
$$

An iterative solver using the generalized minimal residual method (GMRES) is used to find the solution. Once the system is solved, the fluxes in branches are obtained with:

$$
\boldsymbol{\Phi}=\mathbf{P}^{t} \boldsymbol{\Phi}_{\text {loop }}
$$

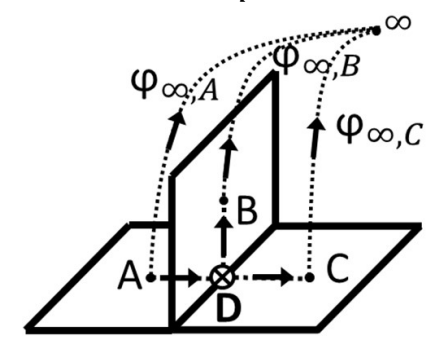

Fig. 7. Equivalent circuit for T shape geometry

The formulation can also be used when T-shape shell geometry are under study. The building of the equivalent circuit needs to put an additional circuit node on the facet sharing more than two elements and to connect the final circuit like presented in Fig. 7. Let us notice that to take into account T-shape geometry is easy with $\mathbf{B}$-formulation presented here but not natural with vector potential formulation [2]. 


\section{POST-PROCESSING}

After the solving, the field in air has to be computed on a point located at a distance $\left|r-r^{\prime}\right|$ from the device. For each element e, the field contribution is:

$$
\boldsymbol{H}_{\text {red }}=-\frac{1}{4 \pi} \boldsymbol{\nabla} \sum_{e=1}^{N_{e}} \int_{S_{e}} \frac{\left(v_{0-}-v\right) \boldsymbol{\nabla}_{s} . \mathbf{B}}{\left|\mathrm{r}-\mathrm{r}^{\prime}\right|} d S_{e}
$$

The computation of the derivative of the Green's kernel in (28) is not trivial when the distance between the point $\mathrm{P}$ and the integration point is very small. Its high singularity $\left(\sim \mathbf{r} / \mathrm{r}^{3}\right)$ would involve using too many Gauss points in order to obtain an accurate result in the context of a numerical integration. This is why our solution consists in the use of analytical expressions for quadrangles and triangles [6]. This method ensures to compute the field close to the shell with a very good accuracy.

\section{RESULTS}

The formulation has been tested on an academic problem whose an analytical solution [7] is known. In Fig. 8, it is a linear ferromagnetic hollow sphere magnetized by a uniform inductor field $\mathbf{H}_{\mathbf{0}}$ oriented in $\mathrm{x}$ direction with a value of $1 \mathrm{~A} / \mathrm{m}$.

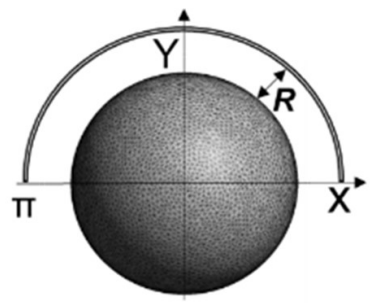

Fig. 8. Hollow sphere composed with 12114 surfaces elements, with a diameter of 200 meters, a thickness of $4 \mathrm{~cm}$ and a relative permeability of 100 . A semicircle is set as a computation support to validate the method.

In order to compare reference and results, the following error criterion is defined:

$$
\mathrm{E}_{\mathrm{i}} \%=\frac{\left\|\mathrm{B}_{\mathrm{i}, \mathrm{ref}}-\mathrm{B}_{\mathrm{i}, \text { Surf }}\right\|}{\max \left\|\mathrm{B}_{\text {ref }}\right\|}
$$

Spatial components of the magnetic induction are computed around the hallow sphere on a semi-circle. The computation support is located on a curve line located at the distance R. This distance will be reduced to $1 \mathrm{~mm}$ to compare the results and the reference very close to the shell.

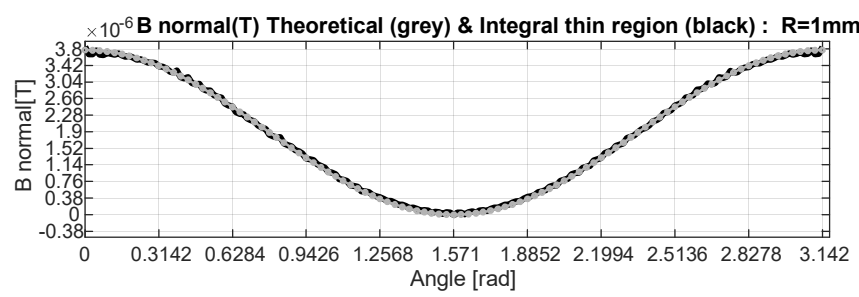

Fig. 9. Normal component of the magnetic induction. Very good match between the analytical solution (dots in gray) and the solution obtained with the integral surface method (black) at $1 \mathrm{~mm}$ from the mesh.

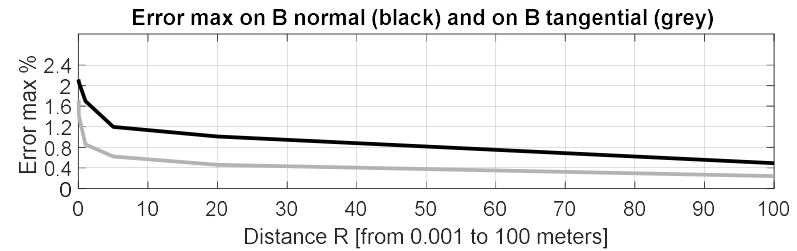

Figure 10: Maximal normal error (black) and maximal tangential error (gray) around the hallow sphere obtained with the integral surface method for several computation distance from the shell.

Fig. 9 and Fig. 10 show the accuracy of the formulation and the analytic post-treatment. The accuracy of the calculated magnetic induction is very good. Although the sphere is meshed by facets (edge's length: 4 meters), the maximal error is under $2.5 \%$ (the average error on the computation line at $1 \mathrm{~mm}$ from the hull is under $1 \%$ ) is obtained extremely close to the hull. As shown in Fig. 11, studies of more complex devices like the magnetic anomaly created by a submarine hull have been performed. This formulation coupled to matrix compression algorithms remains acceptable in terms of computational resources.

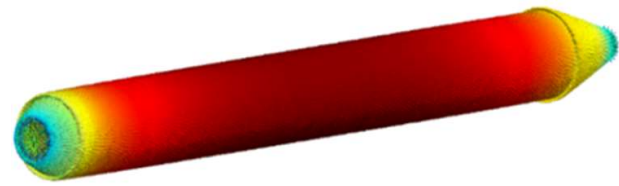

Figure 11: Surface magnetic flux density of a submarine model with 33.298 surfaces elements, 83.245 branches, and 49.947 independent loops, solved in 12 min (i5-6600, 6GB DDR3) with a matrix compression ratio of $89.5 \%$ (ACA).

\section{CONCLUSION}

The VIM has been used to solve a magnetostatic problem with a face interpolation. This method has been adapted to thin magnetic regions and limited to a surface mesh. The method does not require the mesh of the air region and it enables the modeling of shielding problem with a very reasonable computation time. The obtained results are very accurate even if a light mesh is used. The method can be applied to the computation of the magnetic anomaly created by a submarine placed in the earth magnetic field [8].

\section{REFERENCES}

[1] V. Le-Van, G. Meunier, O. Chadebec and J. Guichon, "A Volume Integral Formulation Based on Facet Elements for Nonlinear Magnetostatic Problems," IEEE Trans. Mag., vol. 51, no. 7, pp. 1-6, 2015.

[2] V. Le-Van, G. Meunier, O. Chadebec and J. Guichon, "A Magnetic Vector Potential Volume Integral Formulation for Nonlinear Magnetostatic Problems," IEEE Trans. Mag.,, vol. 52, no. 3, pp. 1-4, 2016.

[3] Harrington, R.F., 1993. Field Computation by Moment Methods. Oxford University Press, USA.

[4] Kalimov, A.G., Svedentsov, M.L., "Three-dimensional magnetostatic field calculation using integro-differential equation for scalar potential," IEEE Trans. Magn. 32, 1996.

[5] A. Canova and M. Repetto, "Integral solution of nonlinear magnetostatic field problems," IEEE Trans. Mag., vol. 37, no. 3, pp. 1070-1077, May 2001.

[6] Rubeck C, Yonnet J-P, Allag H, Delinchant B , and Chadebec O, " Analytical Calculation of Magnet Systems", IEEE Trans. Mag., vol. 49, no. 1 , pp. 144-147, 2013.

[7] Durand, É., 1968. Magnétostatique. Masson et Cie.

[8] X. Brunotte, X., G. Meunier, J-P. Bongiraud, "Ship magnetizations modelling by the finite element method. IEEE transactions on magnetics", IEEE Trans. Mag., vol. 29, no. 2, pp. 1970-1975, 1993. 